\title{
Psychologic Distress and Anxiety in Mothers of Children With Gastroesophageal Reflux Undergoing Antireflux Surgery
}

\author{
Morten Kvello, ${ }^{\mathrm{Z}}$ Tone Lise A vitsland, ${ }^{\S}$ Charlotte K. Knatten, Thomas J. Fyhn, \\ Ulrik Malt, Ragnhild Emblem, and Kristin Bjørnland
}

\begin{abstract}
Objectives: Parents of children with a chronic illness are at risk for impaired psychosocial functioning. Gastroesophageal reflux disease (GERD) is such a disease, and no studies have investigated effects of antireflux surgery on parental psychological distress. The aims of this study were to assess psychological distress and state and trait anxiety in mothers of children with GERD, and to explore possible changes after antireflux surgery.

Methods: Mothers of children referred for antireflux surgery were included in this prospective study. Standardized questionnaires were used to evaluate psychological distress and state and trait anxiety before and 12 months after antireflux surgery.

Results: Of 87 eligible mothers of children with GERD, 62 (71\%) agreed to participate. All children had objectively verified GERD by 24 -hour $\mathrm{pH}-$ monitoring and/or upper gastrointestinal contrast study and unsatisfactory symptom relief of pharmacological treatment. Thirty-one (50\%) mothers returned questionnaires postoperatively. Preoperatively, mothers of children undergoing antireflux surgery reported high levels of psychological distress and state anxiety, and 54\% had scores indicating clinically significant psychological distress. None of the preoperative child characteristics were found to significantly influence maternal psychological distress or state anxiety. Twelve months postoperatively, both psychological distress and state anxiety were reduced.

Conclusions: Mothers of children undergoing antireflux surgery reported reduced levels of psychological distress and state anxiety 12 months after the operation.
\end{abstract}

Key Words: gastroesophageal reflux, gastroesophageal reflux disease, maternal psychosocial functioning, pediatric surgery, prospective trial
What Is Known

Antireflux surgery is effective in treating gastroesoph-ageal reflux disease in children. Chronic childhood disease may influence maternal psychosocial functioning.

\section{What Is New}

Mothers of children with gastroesophageal reflux disease have high levels of psychological distress and anxiety.

Psychological distress and anxiety in mothers decreased 12 months after antireflux surgery.

High levels of parenting stress and distress have been found in parents of children with various chronic diseases (1). For instance, parents of children with congenital heart disease are at risk for psychological distress, anxiety, and depression (2), and similar findings are reported in parents of children with cancer, anorectal malformations, and Hirschsprung disease $(3,4)$. There are also data suggesting that gastroesophageal reflux disease (GERD) in a child may affect parental well-being. A recent study showed significantly higher levels of anxiety and depression in mothers of children with medically treated GERD than in mothers of healthy children (5). Furthermore, a qualitative study of parents of children with GERD reported parental feelings of fear, worry, grief, and depression (6). In most pediatric patients, GERD can be successfully man-aged with conservative and/or pharmacological treatment. In rare cases where symptoms are not effectively relieved, antireflux surgery may be an option (7). Most studies evaluating results after antireflux surgery have focused on complications, length of hospital stay, symptom improvement, and recurrence of GERD (8-10). The effects of antireflux surgery on parental psychological distress are unknown. Therefore, we performed this study to assess psycholog-ical distress and state and trait anxiety in mothers of children undergoing antireflux surgery, hypothesizing that scores would improve after fundoplication. Secondly, we looked for factors that could affect the mothers' psychological distress and state anxiety.

\section{METHODS}

\section{Study Design}

This study is part of a prospective trial, where the primary outcome was to compare recurrence rates of GERD after open and 
laparoscopic Nissen fundoplication (11-13). Patients referred for antireflux surgery at Oslo University Hospital from 2003 to 2009 were eligible for inclusion. Inclusion criteria were age between 0 and 15 years, a diagnosis of GERD defined as troublesome symptoms and/or complications of GERD despite appropriate pharmacological antireflux therapy, and pathologic GER verified by $24-$ hour $\mathrm{pH}$-monitoring and/or upper gastrointestinal contrast study. Exclusion criteria were comorbid conditions or previous surgery hindering laparoscopy, and parents who did not speak Norwegian. Mothers of children included in the trial were eligible for assessment of psychological distress and state and trait anxiety. There were no additional exclusion criteria for mothers.

\section{Ethics}

The Regional Committee for Medical Research Ethics approved the trial. Participants were included after giving written consent. The study is registered in clinicaltrials.gov (NCT01551134).

\section{Preoperative Data}

Child demographics recorded at admittance included age, sex, presence of a gastrostomy, and comorbidity. On the basis of comorbidity, they were classified as having neurologic impairment (NI) or not. NI was defined as a static or progressive, central or peripheral neurological condition associated with intellectual disability and/or functional impairment (14). Severe NI was defined as having 3 or more of the following criteria: cerebral palsy, mostly or exclusively tube-fed, daily epilepsy medications, and nonambu-lance. Non-ambulance was defined as dependency of a wheelchair or a gross motor function classification system score of 4 or 5 in those less than 4 years old (15). Symptoms of GERD and frequency of lower airway infections requiring antibiotic treatment preopera-tively were recorded as dichotomous variables based on parental reports.

\section{Surgical Technique and Follow-up Protocol}

The operative procedure and immediate postoperative management followed a standardized protocol as described previously (11). Briefly, a Nissen fundoplication with hiatal repair was performed in all patients.

For the postoperative follow-up, a visit at Oslo University Hospital or the patient's local hospital was scheduled 6 months postoperatively. This included a clinical examination, as well as upper gastrointestinal contrast and 24-hour $\mathrm{pH}$ studies. In addition, an extensive semi-structured telephone interview was performed 1, 2, and 4 years after surgery. The parents were asked about symp-toms indicating recurrent GERD, possible side effects of operation, the child's general well-being, and overall satisfaction with the operation. The phone interviewers (C.K.K. and T.J.F.) had not been involved in accepting patients for surgery or in the treatment of patients during follow-up.

\section{Assessment of Psychological Distress and State and Trait Anxiety}

To assess psychological distress in mothers of children undergoing antireflux surgery, the General Health Questionnaire 30item version (GHQ-30), a reliable and validated instrument, was used (16). Each of the 30 items is answered on a 4-point Likert scale (0-1$2-3)$, resulting in a total Likert score ranging from 0 to 90 . Higher scores indicate higher levels of distress. GHQ-30 includes 5 subscales: anxiety, well-being, depression, coping failure, and social dysfunction (17). Subscale scores were divided by the number of items in each subscale, giving a range from 0 to 3 . Case scoring (0-0-1-1) was used to identify individuals with clinically significant distress, resulting in a total case score ranging from 0 to 30. A GHQ-30 case score 6 was defined as "'a case',(16).

The State-Trait Anxiety Inventory Form X, a reliable and validated instrument, was used to assess state (STAI-X1) and trait (STAI-X2) anxiety (18). The 12-item version of STAI-X1 was used (19), whilst STAI-X2 consisted of 20 items. Each item is answered on a 4-point Likert scale (1-2-3-4) resulting in a total Likert score ranging from 12 to 48 for STAI-X1 and 12 to 80 for STAI-X2. Higher scores indicate increased anxiety levels.

All questionnaires were validated and in the Norwegian language, and the Norwegian version has previously been used in similar populations (20-22). According to the study protocol, GHQ30 , STAI-X1, and STAI-X2 were administered to the mothers at 3 different time points; preoperatively and 6 and 12 months after surgery. Six months postoperatively, the questionnaires were either given to the mothers during the scheduled hospital follow-up or sent by post if the postoperative follow-up was done at the patient's local hospital. All received questionnaires by post after 12 months. If questionnaires were not returned, a letter was sent to remind the mothers of the questionnaires.

\section{Statistics}

Numerical data are presented as mean and standard deviation (SD) or as median [min-max] if not fitting normal distribution. Categorical data are presented as frequencies and percentages. Analyses was performed comparing patients with NI to patients without NI, and responders to nonresponders. Age was compared using the Mann-Whitney $U$ test as data were not normally distrib-uted, and categorical data were compared using the chi-square test. Preoperative questionnaire scores of postoperative responders and postoperative nonresponders were compared with the independent samples $\mathrm{T}$ test. Questionnaires with more than $20 \%$ of items missing were excluded. In cases with less than $20 \%$ missing items, the mean score of the items filled in, replaced the missing item. A univariate linear regression analysis was performed to identify possible factors influencing preoperative GHQ-30 Likert and STAI-X1 scores. Preoperative and postoperative GHQ-30, STAI-X1, and STAI-X2 scores were normally distributed and compared using the paired samples $\mathrm{T}$ test, calculating mean difference and $95 \%$ confidence intervals. A $\mathrm{P}$ value $<0.05$ was considered statistically significant. All analyses were performed using IBM SPSS Statistics for Windows, Version 24.0. Armonk, NY: IBM Corp., released 2016.

\section{RESULTS}

\section{Study Population}

During the study period, 107 patients underwent a primary Nissen fundoplication. Of these, 20 were excluded or did not want to participate for various reasons (11). The remaining 87 patients were included in the prospective trial, and the mothers of these patients were asked to answer the GHQ-30, STAI-X1, and STAI$\mathrm{X} 2$ questionnaires.

Of the 87 eligible mothers, $62(71 \%)$ returned questionnaires preoperatively. GHQ-30 was missing from one of these mothers, STAI-X2 from another. Only $11(18 \%)$ mothers returned questionnaires 6 months postoperatively, whereas $31(50 \%)$ answered around 12 months after the operation. Due to the low response rate at 6 months, only questionnaires returned at 12 months were used in the postoperative assessment. GHQ-30 was missing from one of these mothers, STAI-X1 from another. The postoperative 


\begin{tabular}{|c|c|c|c|}
\hline & Patients with NI (n 1/4 29) & Patients without NI (n 1/4 33) & P value ${ }^{y}$ \\
\hline Median age $[\min -\max ]$ & $3.0[0.2-15.2]$ & $5.1[0.4-13.6]$ & 0.77 \\
\hline Female & $13(45 \%)$ & $9(27 \%)$ & 0.15 \\
\hline Pre- or perioperative gastrostomy & $28(96 \%)$ & $5(15 \%)$ & $<0.01$ \\
\hline Mostly/only tube fed & $24(83 \%)$ & $4(12 \%)$ & $<0.01$ \\
\hline Vomiting 4-7 d/wk & $17(58 \%)$ & $9(27 \%)$ & 0.01 \\
\hline Retching 4-7 d/wk & $13(45 \%)$ & $1(3 \%)$ & $<0.01$ \\
\hline Regurgitation $4-7 \mathrm{~d} / \mathrm{wk}$ & $14(48 \%)$ & $25(76 \%)$ & 0.03 \\
\hline$>4$ airways infections the last year & $20(69 \%)$ & $15(46 \%)$ & 0.06 \\
\hline
\end{tabular}

Neurological impairment.

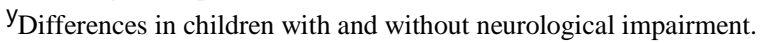

questionnaires were answered median 13.3 months [9.0-19.2] after the operation.

\section{Demographics}

The demographics and GERD symptoms in the children of the 62 mothers included in the preoperative assessment are presented in Table 1. Patients with NI were more likely to have frequent vomiting, retching, and regurgitation than patients without NI. Diagnoses in NI patients included cerebral palsy (n $1 / 4$ 1), various syndromes (n $1 / 46$ ), malformations of the central nervous system (n $1 / 46$ ), and others (n $1 / 46)$. Of the NI patients, $55 \%$ (n $1 / 4$ 16) had severe NI and 45\% (n $1 / 4$ 13) mild NI. In nonNI patients, $88 \%$ (n $1 / 4$ 29) had no other diagnoses than GERD, $6 \%(\mathrm{n} 1 / 42$ ) had esophageal atresia, 3\% (n 1/4 1) had severe kidney disease, and $3 \%(\mathrm{n} 1 / 41)$ had hydrocephalus without NI.

\section{Preoperative Assessment}

Preoperative scores of psychological distress and state and trait anxiety are shown in Table 2. Subscale scores of GHQ-30 showed lower scores in depression compared with other subscales. On the basis of GHQ-30 case scores, 54\% (33/61) of the mothers had a score that indicated clinically significant psychological distress. In a univariate linear regression analysis, neither age of the child, GERD symptoms nor comorbidity influenced the preop-erative GHQ-30 or STAI-X1 scores (Supplemental Table 1, Sup-plemental Digital Content, http://links.lww.com/MPG/B582). Mothers of patients with mild NI had higher GHQ-30 and STAI-

TABLE 2. Preoperative psychological distress (GHQ-30) and state (STAI-X1) and trait (STAI-X2) anxiety of mothers of children with gastroesophageal reflux disease undergoing Nissen fundoplication

\begin{tabular}{|c|c|}
\hline Questionnaire & Mean (SD) \\
\hline GHQ-30 Likert score (n $1 / 461)$ & $35.8(15.0)$ \\
\hline Anxiety $y$ & $1.35(0.67)$ \\
\hline Well-being $y$ & $1.26(0.46)$ \\
\hline Depression ${ }^{y}$ & $0.62(0.57)$ \\
\hline Coping failure ${ }^{\mathrm{y}}$ & $1.24(0.58)$ \\
\hline Social dysfunction ${ }^{y}$ & $1.33(0.56)$ \\
\hline STAI-X12 (n $1 / 462)$ & $24.2(7.4)$ \\
\hline STAI-X2 $2^{Z}$ (n 1/4 61) & $40.5(10.4)$ \\
\hline
\end{tabular}

General Health Questionnaire 30-item version.

y

${ }^{\mathrm{z}}$ State-Trait Anxiety Inventory Form X.
$\mathrm{X} 1$ scores than mothers of patients with severe NI (GHQ-30: $41.0 \mathrm{vs}$ 29.4, P 1/4 0.035, STAI-X1: 27.0 vs 21.1, P 1/4 0.038).

\section{Postoperative Assessment}

All mothers, both responders (n $1 / 4$ 31) and nonresponders (n $1 / 427$, missing $1 / 4$ 4) reported 12 months postoperatively that their expectations of the fundoplication were fulfilled. Ninetyseven percent (n 1/4 30) and 96\% (n 1/4 26) of responders and nonrespon-ders, respectively, reported that their child's well-being had improved after the fundoplication.

There were no significant differences between the children of responders and nonresponders regarding the patients' age, comorbidity, preoperative GERD symptoms, clinical outcomes, or postoperative recurrence of GERD (Table 3). Furthermore, there was no difference in preoperative mean GHQ-30, STAI-X1, or STAI-X2 scores between postoperative responders and nonresponders (GHQ30: 35.3 vs $36.2, \mathrm{P}^{1} / 4$ 0.82, STAI-X1: 23.0 vs 25.3 , P $1 / 4$ 0.22, STAI$\mathrm{X} 2: 39.3$ vs $41.8, \mathrm{P} 1 / 40.35$ ).

The GHQ-30 total and subscales scores, as well as STAI$\mathrm{X} 1$ and STAI-X2 scores of the 31 mothers included in the postoperative assessment are presented in Table 4. There was a statistically significant decrease in both total GHQ-30 Likert score and in subscales measuring anxiety, well-being, and coping failure when comparing pre- and postoperative scores. Furthermore, there was also a statistically significant decrease in STAI-X1 scores when comparing postoperative and preoperative scores. No significant change was found for STAI-X2 scores.

On the basis of postoperative GHQ-30 case-scores, $20 \%$ (n $1 / 4$ 6) of the mothers had scores suggestive of clinically significant psychological distress. Four of these mothers also had preoperative scores suggestive of clinically significant psychological distress. Of these 6 mothers, 3 had children with NI, 2 had children with no other disease than GERD, and 1 had a child with esophageal atresia.

Many parents reported spontaneously during the semi-structured follow-up interviews that domains related to the gastrointestinal system had improved postoperatively. For instance, parents frequently mentioned the relief of no longer having to change the child's clothes several times a day because of vomiting. Other common comments were that they were less stressed during feeds because they no longer were worried that the child would vomit most of the meal.

\section{DISCUSSION}

The present study demonstrates that mothers of children referred for antireflux surgery have high levels of psychological distress and state anxiety when standardized and validated questionnaires are applied. Importantly, these scores decreased 
TABLE 3. Comparison of demographics, preoperative data, and clinical outcomes in children of mothers who did and did not take part in the postoperative assessment

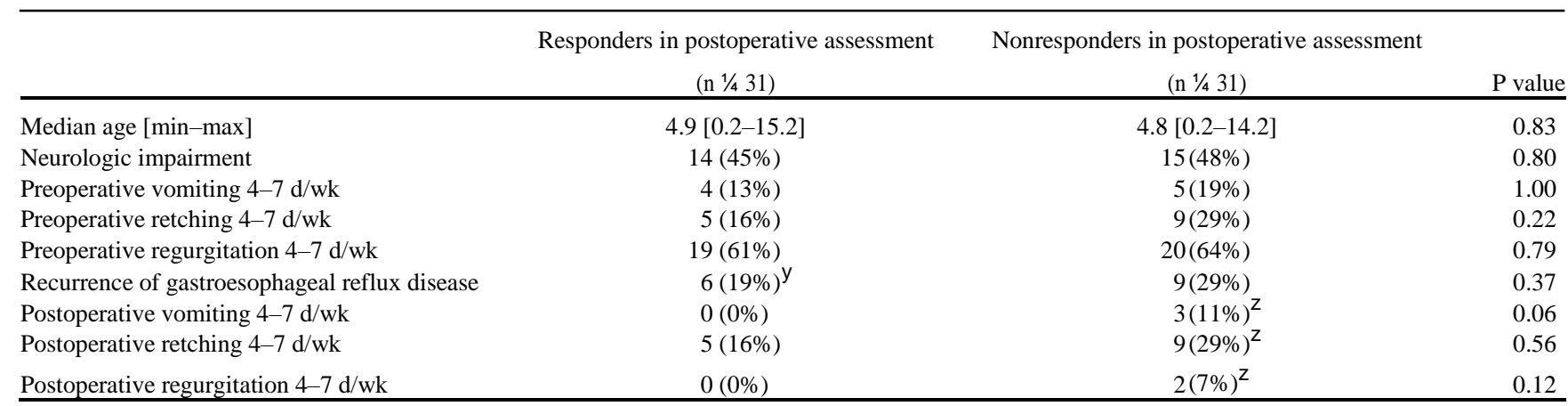

Differences in children of responders and nonresponders.

${ }^{\mathrm{y}}$ Two children were treated conservatively, and 4 had a redo fundoplication. All 6 children experienced initial improvement of gastroesophageal reflux disease symptoms after the operation, and in 5 of these, the symptoms of recurrent gastroesophageal reflux disease occurred after the mothers had answered postoperative questionnaires. In 1 child, undergoing a redo Nissen fundoplication for a symptomatic hiatal hernia 11 months postoperatively, the patient had symptoms of the hiatal hernia at the time when the mother answered the postoperative questionnaire.

${ }^{\mathrm{N}} \mathrm{N}^{1 / 4} 27$, missing $1 / 44$.

significantly after antireflux surgery, indicating that antireflux surgery may be beneficial not only for the child but also for the mothers as well. This study is the first to examine the effects of fundoplication on psychological distress and state anxiety in a population of mothers of children undergoing antireflux surgery.

The children in this study underwent antireflux surgery because conservative and medical treatment did not efficiently relieve troublesome GERD symptoms. Qualitative studies in parents of children with GERD report that the child's disease causes stress and impaired sleep, as well as worry and frustration (23). In addition, the families' social life and well-being may be influenced, as well as the parents' ability to have a full-time job (6). A case-control study investigating emotional well-being in mothers of medically treated non-NI children, found higher levels of depres-sion and anxiety compared with mothers of healthy children (5). In line with this, we found elevated levels of both psychological distress and state anxiety in mothers of children referred for fundoplication. Notably, over $50 \%$ of the mothers had scores suggesting clinically significant psychological distress. The mothers in this study had higher scores of psychological distress than mothers of children undergoing gastrostomy placement (mean GHQ-30 Likert score 35.8 vs 29.8) (22), and the scores are comparable to those of parents of children with severe meningococcal disease and cancer $(24,25)$. Subscale analysis of the GHQ-30 scores showed increased anxiety, well-being and coping failure scores, and lower scores for depression. This implies that on a group level, the increased scores in mothers were likely to be a sign of stress from the child's disease, rather than severe psychiatric illness. This assumption is supported by findings of levels of trait anxiety within the normal range (18).

Twelve months after the Nissen fundoplication, we found a significant decrease in both psychological distress and state anxiety in the mothers. In line with these results, the patients experienced relief of GERD symptoms, and parents were satisfied with the result of the fundoplication $(12,13)$. It seems that relief of the child's GERD symptoms improved maternal emotional well-being. The spontaneous comments from mothers during the semi-structured interviews postoperatively further support the positive effect of the fundoplication on the mothers. Thus, the results support our hypothesis that mothers are influenced by the children's disease, and that when GERD is efficiently treated, maternal levels of distress and state anxiety are reduced (25). In line with our findings, Engelmann et al (26) reported improvement in parent-centered domains of healthrelated quality of life (HRQOL) 6 months after fundoplication. Two other studies using self-designed question-naires also reported a positive impact on parental quality of life after

TABLE 4. Psychological distress (GHQ-30) and state (STAI-X1) and trait (STAI-X2) anxiety in mothers of children before and after Nissen fundoplication

\begin{tabular}{|c|c|c|c|c|}
\hline Questionnaire & Preoperatively (mean) & Postoperatively (mean) & Mean difference $(95 \% \mathrm{CI})$ & $P$ value \\
\hline GHQ-30 Likert score (n 1/4 30) & 35.6 & 26.6 & $9.0(2.8-15.2)$ & $<0.01$ \\
\hline Anxiety $y$ & 1.37 & 0.92 & $0.44(0.17-0.71)$ & $<0.01$ \\
\hline Well-being ${ }^{y}$ & 1.25 & 0.96 & $0.29(0.07-0.52)$ & 0.01 \\
\hline Depression ${ }^{y}$ & 0.66 & 0.59 & 0.05 ( 0.12 to 0.22$)$ & 0.53 \\
\hline Coping failure $^{\mathrm{y}}$ & 1.23 & 0.95 & $0.28(0.04-0.52)$ & 0.02 \\
\hline Social dysfunction ${ }^{y}$ & 1.22 & 0.99 & $0.23(0.01$ to 0.47$)$ & 0.05 \\
\hline STAI-X1 (n 1/4 30) & 23.1 & 19.2 & $3.9(1.3-6.4)$ & $<0.01$ \\
\hline STAI-X2Z (n 1/4 31) & 39.3 & 38.2 & $1.1(2.0$ to 4.2$)$ & 0.48 \\
\hline
\end{tabular}

General Health Questionnaire 30-item version.

$\mathrm{y}_{\text {Subscale of GHQ-30. }}$

${ }^{\mathrm{z}}$ State-Trait Anxiety Inventory Form X. 
fundoplication (27,28). Contrasting these finding that antireflux surgery may improve parental emotional well-being, is a study from Srivastava et al (29) who found no improvement in parenting stress as measured with the Parenting Stress Index and HRQOL 1 month after surgery. The authors hypothesized that the lack of change was because of the short time interval between assessments.

Patients with NI presented with more frequent symptoms of GERD, and about half of NI patients were classified with severe NI. We did not find higher preoperative psychological distress in mothers of children with NI and GERD than in mothers of non-NI children with GERD. This finding contrasts other studies reporting that parents of NI children have impaired emotional well-being compared with parents of non-NI children $(30,31)$. There are several explanations for these contrasting observations. Established coping mechanisms in parents of NI children may explain the lack of difference between these 2 groups of parents (32). As all these children had so severe GERD symptoms that surgery was considered necessary, it is possible that the mothers' concern for GERD outweighed that of NI. Furthermore, we did not find that mothers of those with severe NI scored higher than mothers of children with mild NI. This supports the finding that GERD was a significant stressor for these mothers.

A major advantage of this study was the use of standardized and validated questionnaires, as well as the prospective and longitudinal study design. Furthermore, the clinical status of the children was very well documented and can be related to results from the questionnaires on maternal psychological distress and state anxiety. As we only included children with objectively verified GERD and unsatisfactory effect of medical treatment, results from this study may be generalizable to other pediatric GERD popula-tions referred for antireflux surgery.

There are, however, several limitations of this study. The first set of questionnaires assessing psychological distress and state and trait anxiety was answered during the hospital stay when the operation was performed, and hospitalization and surgery may have served as a stressor for the parents (33). Another limitation is that a significant number of participants did not return postoperative questionnaires. Low response rates represent a risk for nonre-sponse bias. The drop-out analysis, however, showed no significant difference in either demographics, pre- and postoperative GERD symptoms or parental satisfaction between responders or nonre-sponders. Furthermore, the preoperative psychological distress and state anxiety scores in the mothers were similar. Thus, it is reasonable to assume that the conclusions would not be different if more mothers had returned the questionnaires. Another disad-vantage of the study design is that possible confounding factors, such as mothers' sociodemographic status, major life events (eg, divorce, disease), or changes in the child's underlying condition were not assessed. Such information would have strengthened the study. Lastly, a few patients got a gastrostomy inserted concomi-tantly with the fundoplication. As gastrostomy placement has been shown to improve maternal psychological distress, some of the effect contributed to the fundoplication, may have been caused by the gastrostomy (22).

\section{CONCLUSIONS}

To conclude, we have shown that mothers of children referred for antireflux surgery have high levels of psychological distress and state anxiety before the operation. Secondly, we found that successful antireflux surgery improved maternal psychological distress and state anxiety. Even though improvement of maternal psychosocial functioning should not be an indication for antireflux surgery in a child, it is an important outcome measure of the surgical treatment.

\section{REFERENCES}

1. Cousino MK, Hazen RA. Parenting stress among caregivers of children with chronic illness: a systematic review. J Pediatr Psychol 2013;38:809-28.

2. Kolaitis GA, Meentken MG, Utens EMWJ. Mental health problems in parents of children with congenital heart disease. Front Pediatr 2017;5:1-7.

3. Ljungman L, Cernvall M, Grönqvist H, et al. Long-term positive and negative psychological late effects for parents of childhood cancer survivors: a systematic review. PLoS One 2014;9:e103340.

4. Witvliet M, Sleeboom C, de Jong J, et al. Anxiety and quality of life of parents with children diagnosed with an anorectal malformation or Hirschsprung disease. Eur J Pediatr Surg 2014;24:70-4.

5. Karacetin G, Demir T, Erkan T, et al. Maternal psychopathology and psychomotor development of children with GERD. J Pediatr Gastroenterol Nutr 2011;53:380-5.

6. Kim J, Keininger DL, Becker S, et al. Simultaneous development of the Pediatric GERD Caregiver Impact Questionnaire (PGCIQ) in American English and American Spanish. Health Qual Life Outcomes 2005;3:5.

7. Rosen R, Vandenplas Y, Singendonk M, et al. Pediatric gastroesophageal reflux clinical practice guidelines: joint recommendations of the North American Society for Pediatric Gastroenterology, Hepatology, and Nutrition (NASPGHAN) and the European Society for Pediatric Gastroenterology, Hepatology, and Nutrition (ESPGHAN). J Pediatr Gastroenterol Nutr 2009;49:498-547.

8. Jancelewicz T, Lopez ME, Downard CD, et al. Surgical management of gastroesophageal reflux disease (GERD) in children: a systematic re-view. J Pediatr Surg 2017;52:1228-38.

9. Diaz DM, Gibbons TE, Heiss K, et al. Antireflux surgery outcomes in pediatric gastroesophageal reflux disease. Am J Gastroenterol 2005;100:1844-52.

10. Kristensen C, Avitsland T, Emblem R, et al. Satisfactory long-term results after Nissen fundoplication. Acta Paediatr 2007;96:702-5.

11. Knatten CK, Fyhn TJ, Edwin B, et al. Thirty-day outcome in children randomized to open and laparoscopic Nissen fundoplication. J Pediatr Surg 2012;47:1990-6.

12. Fyhn T, Knatten C, Edwin B, et al. Randomized controlled trial of laparoscopic and open Nissen fundoplication in children. Ann Surg 2015;261:1061-7.

13. Knatten CK, Kvello M, Fyhn TJ, et al. Nissen fundoplication in children with and without neurological impairment: a prospective cohort study. J Pediatr Surg 2016;51:1115-21.

14. Barnhart DC, Hall M, Mahant S, et al. Effectiveness of fundoplication at the time of gastrostomy in infants with neurological impairment. JAMA Pediatr 2013;167:911-8.

15. Rosenbaum PL, Palisano RJ, Bartlett DJ, et al. Development of the gross motor function classification system for cerebral palsy. Dev Med Child Neurol 2008;50:249-53.

16. Goldberg D, Williams P. A User Guide to the General Health Questionnaire. Windsor, UK: NFER-NELSON; 1988.

17. Huppert FA, Walters DE, Day NE, et al. The factor structure of the General Health Questionnaire (GHQ-30). A reliability study on 6317 community residents. Br J Psychiatry 1989;155:178-85.

18. Spielberger CD, Gorsuch RL, Lushene RE. STAI Manual for the State-Trait Anxiety Inventory. Palo Alto, CA: Consulting Psychologists Press; 1970.

19. Malt UF, Olafsen OM. Psychological appraisal and emotional response to physical injury: a clinical, phenomenological study of 109 adults. Psychiatr Med 1992;10:117-34.

20. Malt UF, Mogstad TE, Refnin IB. Goldberg's General Health Questionnaire. Tidsskr Nor Laegeforen 1989;109:1391-4.

21. Misund AR, Nerdrum $\mathrm{P}, \mathrm{Bra}^{\circ}$ ten $\mathrm{S}$, et al. Long-term risk of mental health problems in women experiencing preterm birth: a longitudinal study of 29 mothers. Ann Gen Psychiatry 2013;12:33.

22. Avitsland TL, Faugli A, Pripp AH, et al. Maternal psychological distress and parenting stress after gastrostomy placement in children. J Pediatr Gastroenterol Nutr 2012;55:562-6.

23. Acierno SP, Chilcote HC, Edwards TC, et al. Development of a quality of life instrument for pediatric gastroesophageal reflux disease: qualitative interviews. J Pediatr Gastroenterol Nutr 2010;50:486-92. 
24. Ehrlich TR, Von Rosenstiel IA, Grootenhuis MA, et al. Long-term psychological distress in parents of child survivors of severe meningococcal disease. Pediatr Rehabil 2005;8:220-4.

25. Stam H, Grootenhuis MA, Brons PP, et al. Health-related quality of life in children and emotional reactions of parents following completion of cancer treatment. Pediatr Blood Cancer 2006;47:312-9.

26. Engelmann C, Gritsa S, Ure BM. Impact of laparoscopic anterior 270 degrees fundoplication on the quality of life and symptoms profile of neurodevelopmentally delayed versus neurologically unimpaired children and their parents. Surg Endosc 2010;24:1287-95.

27. Pacilli M, Eaton S, McHoney M, et al. Four year follow-up of a randomised controlled trial comparing open and laparoscopic Nissen fundoplication in children. Arch Dis Child 2014;99:516-21.

28. O'Neill J, O'Neill P, Goth-Owens T, et al. Care-giver evaluation of antigastroesophageal reflux procedures in neurologically impaired children: what is the real-life outcome? J Pediatr Surg 1996;31:375-80.
29. Srivastava R, Downey EC, Feola P, et al. Quality of life of children with neurological impairment who receive a fundoplication for gastroeso-phageal reflux disease. J Hosp Med 2007;2:165-73.

30. Emerson E. Mothers of children and adolescents with intellectual disability: social and economic situation, mental health status, and the self-assessed social and psychological impact of the child's difficulties. J Intellect Disabil Res 2003;47 (Pt 4-5): 385-99.

31. Oelofsen N, Richardson P. Sense of coherence and parenting stress in mothers and fathers of preschool children with developmental disability. J Intellect Dev Disabil 2006;31:1-12.

32. Taanila A, Syrja“la“ L, Kokkonen J, et al. Coping of parents with physically and/or intellectually disabled children. Child Care Health Dev 2002;28:73-86.

33. Whelan TA, Kirkby RJ. Parent adjustment to a child's hospitalisation. J Fam Stud 2000;6:46-64. 